R E V I S S T A D D E E E S T U D I O S

\title{
Internacionalización y política económica en América Latina
}

Sebastián Edwards

\section{INTRODUCCIÓN}

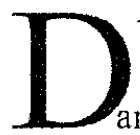

urante los últimos cuarenta años el panorama económico latinoamericano ha cambiado en forma notable. Para alguien instalado en Santiago de Chile a mediados de la década de los años sesenta -época en que fue fundado el Instituto de Estudios Internacionales-, hubiera sido extremadamente difícil predecir que en tan solo cuatro décadas prácticamente en toda la región dominaría el sistema de mercado. Más difícil hubiera sido imaginar que empresas públicas de la importancia y simbolismo de YPF en la Argentina y CAP en Chile, entre muchas otras, estarían en manos de accionistas privados.

Los debates económicos de hace cuarenta años se centraban en temas que hoy parecen irrelevantes o simplemente exóticos. Recuerdo mi propia experiencia como estudiante primerizo en la Escuela de Economía de la Universidad de Chile -que entonces se encontraba en la vieja casona de la calle República-, dónde nos pasábamos horas estudiando los aspectos teóricos y prácticos del «socialismo comunitario» a la sazón imperante en la Yugoslavia del Mariscal Tito. Ninguno de nosotros se imaginaba que al llegar a la cincuentena no existirían ni el sistema comunitario ni el país que lo había hecho famoso. Vuelvo a mis días en la calle República-los primeros años de la década de los años setenta-y no sé si reírme o avergonzarme al recordar el fervor con que leíamos y analizábamos, o mejor dicho tratábamos de analizar, unos pésimos textos marxistas en peores traducciones. Los más sabihondos repetían de memoria largos pasajes de El 18 de Brumario de Luis Bonaparte, y de La Sagrada Familia, y los más tecnificados estudiábamos obtusas técnicas de planificación, las que nuestros profesores nos aseguraban ayudarían a resolver los problemas económicos de la región y del mundo entero. Y cuando 
algún estudiante preguntaba por los precios y su rol en la asignación de recursos, el profesor lo miraba como si fuera un tarado o algo peor. Después explicaba, con marcado aire de impaciencia, que los precios no eran otra cosa que la solución dual del programa de optimización que se llevaba a cabo en la oficina de planificación. Así de simple: todo lo que había que hacer era multiplicar dos matrices -claro, eran (o debían ser), unas matrices gigantes-y luego invertir la matriz resultante. $Y$ ipresto! En una oficina cualquiera se solucionaba el problema de la asignación de recursos de un país de una enorme complejidad. Luego, se calculaba el dual y se obtenían los «verdaderos» precios. Por verdaderos se entendía los «precios de cuenta» o «precios sociales», precios que en teoría reflejaban la verdadera escasez de bienes. Precios muy distintos de los precios capitalistas que, según nos aseguraba el profesor, usaban las empresas para explotar a los pobres, desprevenidos e ingenuos consumidores.

Lo que resulta particularmente curioso mirado desde la perspectiva actual, es que aun los economistas que creían que al mercado le correspondía un rol importante, tenían una visión más bien tímida de lo que debía o podía hacerse en materia de política económica. Quizás la forma más clara de ilustrar este punto es releyendo el programa económico que un grupo de economistas de oposición -conocido como los Chicago Boys- prepararon en Chile en 1973, durante el gobier- no del doctor Salvador Allende. Las propuestas de apertura externa hablan de aranceles diferenciados, a niveles de hasta $35 \%^{1}$. Ni pensar en aranceles parejos al $6 \%$ como los que Chile tiene actualmente.

\section{Los economistas tenían una visión tímida de lo que podía hacerse en materia de política económica.}

Sin embargo, hay un tema que, al menos entre algunas mentes visionarias, ha estado presente en el debate desde esos años. Recuerdo que fue en 1969 , cuando siendo aún estudiante de secundaria, asistí por primera vez a una reunión académica sobre política económica. Se trataba de un seminario organizado por el Instituto de Estudios Internacionales que se desarrolló en Viña del Mar, y que exploraba los beneficios que le traería a Chile una política que mirara hacia el $\mathrm{Pa}$ cífico, incluyendo especialmente los países de Oceanía y Asia. La verdad es que no entendí mayormente lo que los expositores dijeron en esa oportunidad, pero tuve dos intuiciones. Pensé que sería interesante ser un economista profesional, y también pensé que la idea de orientarnos hacia el Pacífico, ampliamente entendido, hacía mucho sentido; mucho más, de hecho, que concentrarnos en los países del Pacto Andino (una especie de Pacífico restringido y limitado), tema que en esa época empezaba a ponerse de moda.

1 Ver El Ladrillo: Bases de la Política Económica del Gobiemo Militar Chileno, Centro de Estudios Públicos, Santiago de Chile, 1992. 
Aunque las tasas de inflación se redujeron, los niveles de pobreza continúan siendo deplorables.

A pesar de los cambios en la visión y las políticas económicas en los últimos cuarenta años, alcanzar una tasa de crecimiento sosteniblemente elevada continúa siendo una meta elusiva en América Latina. Los primeros 12 años de las reformas modernizadoras del llamado Consenso de Washington dieron escasos resultados en términos de crecimiento. Es cierto que las tasas de inflación se redujeron en forma importante en todos los países de la región, pero también es cierto que las condiciones sociales -incluyendo los niveles de pobreza-continúan siendo deplorables en la gran mayoría de ellos. En 1997-1998, años de las crisis financieras en Asia y Rusia, la mayor parte de América Latina entró en un período de bajísimo crecimiento. Solo en los últimos dos años -a partir de 2004-ha habido una recuperación económica importante, recuperación que se ha producido a partir de la enorme mejoría de los precios de exportación de la región y del crecimiento sin precedentes de la economía mundial. De hecho, en su conjunto, los años 2004 y 2005 corresponden al mejor bienio, en términos de desempeño económico, registrado por la región desde los años sesenta. Más aun, todo indica que el trienio 2004-2006 será el de mayor crecimiento económico en un período igualmente largo. Lo que hace que esto sea particularmente importante es que este crecimiento acelerado ha tenido lu- gar en un contexto en que la región ha podido mantener la estabilidad macroeconómica. A partir de 2004, América Latina ha podido evitar el estallido de grandes crisis de balanza de pagos y/o inflaciones aceleradas. Las últimas tres grandes crisis se produjeron a principios de la década de 2000 en Argentina, la República Dominicana y Uruguay.

Estos sucesos platean una importante pregunta: ¿Está América Latina finalmente experimentando el despegue económico que hasta ahora le ha sido tan esquivo? ¿Estaremos, quizás, en los albores de una nueva era? ¿Es este el principio, tal vez, de la «década de América Latina»? Estos son los temas que aborda este artículo.

\section{El Consenso de Washington: UN INVENTO}

El mediocre comportamiento de las economías latinoamericanas en el período 1997-2003 generó una creciente decepción entre los votantes, que empezaron a cuestionar las reformas económicas introducidas a principios de la década de los años noventa. Prácticamente en toda la región hubo críticas al llamado Consenso de Washington, o conjunto de reformas modernizadoras.

Según los críticos, estas reformas fueron concebidas y diseñadas en Washington a fines de los años ochenta por funcionarios del Tesoro estadounidense, del Banco Mundial y del Fondo Monetario Internacional -de ahí el nombre de Consenso de Washington. Más aún, estas reformas habrían sido impuestas a los paí- 
ses de la región, y habrían carecido de un componente social, ignorando la necesidad de mejorar las condiciones de vida de los segmentos más pobres de la población ${ }^{2}$.

\section{El Banco Mundial se mostró escéptico} respecto de la forma del sistema de pensiones en Chile.

Esta visión sobre las reformas y el llamado Consenso de Washington es extremadamente simplista, e ignora aspectos centrales de la historia económica reciente de América Latina. Para empezar, las ideas de las reformas no fueron desarrolladas ni en Washington ni por funcionarios del Tesoro o de las organizaciones multilaterales. Además, y como lo señalan múltiples documentos de la época, sus objetivos directos eran producir mejoras en las condiciones sociales de la población ${ }^{3}$. Las reformas de los años ochenta y noventa fueron concebidas y diseñadas por economistas latinoamericanos que percibían que el antiguo modelo de crecimiento -basado en el proteccionismo, la expansión fiscal, y las regulaciones del Estado- estaba enfrentando una crisis terminal. Estas personas entre los que se encontraban Domingo Cavallo en Argentina y Pedro Aspe en México- tenían ideas que iban mucho más allá de las entonces imperantes en los círculos burocráticos de Washington ${ }^{4}$. De hecho, es bien sabido que el FMI miró con preocupación la rápida apertura económica de México, y que se opuso al plan de convertibilidad argentino de 1991. De otro lado, inicialmente el Banco Mundial tuvo una actitud escéptica respecto de la reforma del sistema de pensiones en Chile ${ }^{5}$.

Más que por Washington, la adopción de las reformas en un país tras otro, estuvo influenciada por una serie de acontecimientos hispanoamericanos. En primer lugar, las políticas del gobierno español de Felipe González fueron sumamente influyentes. Dos medidas significativas, adoptadas en los tempranos ochenta, fueron particularmente importantes: la incorporación de España a la OTAN en 1982 -proceso en el que González tuvo que imponerse a miembros de su propio partido, que creían que España no debía sumarse al tratado militar-y las reformas económicas de reconstrucción industrial de la mitad de los años ochenta que modernizaron la economía española. En segundo término, el ejemplo de Chile -el exitoso pionero de las reformas en América Latina- cobró gran importancia a partir de 1990. Hasta ese momento los líderes democráticos de la región habían rechazado la aplicabilidad de la experiencia chilena, debido a que ella se había desarrollado bajo un régimen dictatorial. Desde 1990, sin embargo, el gobierno democrático del pre-

2 Esta crítica la repite, entre otros, Joseph E. Stiglitz en Globalization and its Discontent, Nueva York, W.M. Norton, 2002.

3 Williamson, John, The Political Economy of Policy Reform, Institute for International Economics, 1994.

4 Cavallo, Domingo, Volver a crecer, Buenos Aires, Sudamericana-Planeta, 1984.

5 Para una discusión más completa, véase Edwards Sebastián, Crisis and Reform in Latin America: From Despair to Hope, Oxford, Oxford University Press, 1995. 
sidente Patricio Aylwin no solo ratificó las políticas de libre mercado, sino que profundizó muchas de ellas, incluyendo la apertura económica. Al mismo tiempo, la administración Aylwin aumentó el gasto social y enfatizó las políticas anti-pobre$\mathrm{za}$, dándole legitimidad a las reformas. Todo ello contribuyó a que el caso chileno se transformara en un referente sobre el tipo de orientación económica a seguir, $y$ sirviera como guía a los países que emprendían el proceso de modernización ${ }^{6}$.

A estos dos factores hispanoamericanos hay que sumarles la caída del muro de Berlín y el final de la Guerra Fría, como detonantes que convencieron a un grupo de políticos y economistas de la región de la necesidad de emprender el largo camino de las reformas. Una vez que se inició el proceso, los organismos multilaterales de Washington proporcionaron ayuda, tanto técnica como financiera, para la aplicación de las políticas modernizadoras. Pero el que hayan ayudado no significa que hayan sido estos organismos quienes primero pensaron en este proceso reformista, ni mucho menos que hayan impuesto las reformas a los gobiernos de la región.

Todo esto significa que la reacción anti-reformas que se ha visto en una serie de países a partir de 1998 -reacción que se ha manifestado en la elección de líderes con una retórica crecientemente neopopulista- no es, en realidad, una reacción contra ideas foráneas, sino más bien un rechazo a un concepto de desarrollo elaborado por economistas autóctonos.

\section{En Argentina, las reformas económicas de los años noventa no significaron el fin de la crisis.}

\section{ARgENTINA 2001-2002: ¿LA ÚLTIMA GRAN CRISIS LATINOAMERICANA?}

A lo largo de su historia moderna, América Latina ha vivido una multitud de crisis cambiarias y de balanza de pagos. Por ejemplo, durante los primeros diez años de existencia del Instituto de Estudios Internacionales, hubo profundas crisis cambiarias en Argentina, Bolivia, Brasil, Chile, Colombia, Costa Rica, Ecuador, México, Perú, Uruguay y Venezuela ${ }^{7}$.

Las reformas económicas de los años noventa no significaron el fin de las crisis. Al contrario, puede argumentarse que algunas de las crisis de los años noventa y de los 2000 han sido de las más costosas y profundas de la historia económica latinoamericana. Todo comenzó con la gran crisis mexicana de 1994-1995, en la que el peso se devaluó más de $100 \%$, poniendo en riesgo la estabilidad del sistema financiero internacional. A esta le siguieron las crisis brasilera y ecuatoriana. Pero de todas las crisis de la era post-reformas, ninguna ha sido tan espectacular y trau-

6 Con el tiempo, sin embargo, Chile ha generado ciertos anticuerpos entre los políticos de algunos países de la región.

7 Ver Edwards, Sebastián, Real Exchange Rates, Devaluation and Adjustment: Exchange Rate Policies in Developing Countries, Cambridge, Mass. The MIT Press, 1989. 
mática como la crisis argentina de 20012002.

\section{La ley de convertibilidad argentina creó un sistema de caja de conversión moderno.}

En abril de 1991, y para combatir la hiperinflación y la inestabilidad macroeconómica, el gobierno del Presidente Carlos Menem diseñó y aprobó la «Ley de Convertibilidad». La característica central de esta política fue fijar el valor de la moneda (originalmente el austral, y más tarde el peso) al dólar estadounidense ${ }^{8}$. El valor inicial fue de 10.000 australes por dólar estadounidense; cuando se introdujo el peso en 1992, la relación fue de un peso un dólar. De acuerdo con la ley de convertibilidad, el Banco Central debía usar la política monetaria estrictamente para defender la paridad cambiaria. Según esta ley, el Banco Central no podía otorgar préstamos al gobierno ni emitir pesos sin un estricto respaldo en moneda extranjera. El tipo de cambio fijo debía proveer el tan necesitado control sobre la creación de moneda y, de esta forma, poner frenos a la inflación que se había tornado descontrolada. Desde el punto de vista de la política económica, la ley de convertibilidad creó un sistema de caja de conversión moderno?.
En conjunción con la ley de convertibilidad, el gobierno argentino introdujo un paquete de reformas estructurales. En particular, la vasta mayoría de las empresas públicas fueron privatizadas, el mercado de capitales fue reformado, se relajaron parcialmente muchas regulaciones sobre las inversiones, la economía se abrió parcialmente, se introdujo una reforma tributaria y en parte se privatizó el sistema de seguridad social ${ }^{10}$. Estas reformas tuvieron como objetivo atraer inversión extranjera y aumentar la productividad y el ahorro interno y, por tanto, generar un aumento en la tasa de crecimiento económico.

El gobierno argentino, sin embargo, no aplicó -o solo aplicó parcialmente- una serie de políticas requeridas para apoyar y dar solidez y credibilidad al régimen cambiario de conversión. En particular, no se puso en práctica una política fiscal anticíclica, no se introdujeron medidas encaminadas a flexibilizar el mercado laboral, y la economía no se abrió lo suficiente al resto del mundo. Como consecuencia de ello, en los años noventa Argentina desarrolló un sistema económico altamente vulnerable a las perturbaciones externas y al contagio financiero proveniente de crisis producidas en otros países.

A partir de 1998 Argentina -así como el resto de la región-sufrió una serie de perturbaciones externas -incluyendo una

8 En 1992 el peso reemplazó el austral. El valor del nuevo peso se fijó en 10.000 australes. La creación del peso no modificó el tipo de cambio fijo.

9 Corden, W. Max, «Argentina's Great Currency Board Experiment,» en W.M. Corden, Too Sensational: On the Choice of Exchange Rate Regimes, Cambridge, Mass. The MIT Press, 2002.

10 Banco Mundial, «Argentina, the Convertibility Plan: Assessment and Potential Prospects», 1996. 
caída transitoria de la relación de intercambio-que desembocaron en una de las mayores crisis de la historia del país. A fines de 2001 Argentina congeló los depósitos bancarios y repudió su deuda externa. A principios de 2002 el peso fue devaluado en forma asimétrica y una serie de contratos internacionales fueron alterados en forma unilateral. El nivel de actividad de la economía se desplomó, generando un altísimo nivel de desempleo" .

\section{La crisis argentina ha generado cambios importantes en las políticas económicas de la región.}

Una de las consecuencias de la crisis argentina es que ha generado importantes cambios en las políticas económicas de la región. Tres de estos cambios son particularmente notables: en primer lugar, prácticamente todos los países han dejado de lado los tipos de cambio fijo, y han adoptado sistemas de flexibilidad cambiaria. Ello les ha permitido absorber mejor las perturbaciones externas, y les ha evitado tener que enfrentar la dificil situación de defender una paridad cambiaria muchas veces indefendible. En segundo término, se ha llegado a la convicción de que la política fiscal debe ser contracíclica. Vale decir, durante los años de bonanza hay que acumular reservas fiscales, las que luego pueden utilizarse durante los años de desaceleración económica y de conmociones externas. Y ter- cero, se ha reconocido que si los agentes económicos-empresas, familias o gobierno- se encuentran endeudados en moneda extranjera, una devaluación de la moneda puede resultar devastadora, ya que genera un enorme aumento en el valor de esas deudas en moneda nacional. Por ello, en los últimos años la mayoría de los países han procurado sustituir deuda en moneda extranjera por deuda en moneda local.

Estas políticas, nacidas como lecciones de la crisis Argentina, le han dado nueva robustez y fortaleza a las economías latinoamericanas. Como consecuencia de ello, se ha reducido mucho la probabilidad de que la región vuelva a sufrir crisis de esa magnitud - 0 de la magnitud de la crisis mexicana de 1994-1995-. Desde luego, ello no significa, que no haya probabilidades de que se produzca una mega crisis, pero sí que esta probabilidad es hoy día mucho menor que hace tan solo cinco años. Este es un desarrollo muy importante para la región, desarrollo que debe entenderse en toda su magnitud. Una de las causas fundamentales del deficiente desempeño económico de los países latinoamericanos a lo largo de los años han sido, precisamente, las crisis recurrentes. Cada vez que se produce una crisis mayúscula, la tasa de crecimiento per cápita cae entre $4 \%$ y $5 \%$. Evitar estas crisis ayudará significativamente a la región a mejorar su desempeño económico a largo plazo.

11 Si la Argentina hubiese implementado las políticas de apoyo al régimen de convertibilidad discutidas más arriba, la crisis -incluyendo la devaluación del peso- se hubiese evitado. 
IV. Y EL CRECIMIENTO ECONÓMICO ¿QUÉ?

Una cosa es evitar los retrocesos económicos generados por las grandes crisis, y otra lograr tasas de crecimiento sosteniblemente elevadas a través del tiempo. Ahora que América Latina ha aprendido -o parece haber aprendidocómo evitar grandes crisis, el desafío es lograr un crecimiento lo bastante elevado como para reducir en forma efectiva los niveles de pobreza, y mejorar tanto las condiciones sociales de la población como la distribución del ingreso.

\section{Tras años de estudios, los economistas tienen buena idea de cuáles son los factores determinantes del crecimiento económico.}

Después de años de investigaciones -y de la publicación de cientos de libros y artículos académicos-, los economistas tienen una buena idea de cuáles son los principales factores determinantes del crecimiento económico. En efecto, el crecimiento económico sostenido es el resultado de un proceso complejo, en que se combinan (de manera no lineal), tres factores fundamentales: 1) La acumulación de capital (tanto capital físico como capital humano); 2) La utilización de la mano de obra y 3) Los incrementos de la productividad.

Además de estos factores puramente económicos, tres elementos más generales explican las diferencias en las tasas de crecimiento que hemos observado a través de la historia. En primer lugar, la geografía es importante. Los países que se encuentran más cerca de los grandes mercados -entendiendo la «cercanía» en un sentido amplio- tienen una ventaja sobre los países más distantes. En segundo término, la cultura de un país juega un rol fundamental. Aquí, nuevamente, hay que entender cultura en forma amplia, incluyendo las tradiciones, las instituciones (tanto del Estado como de la sociedad civil), y las actitudes frente al trabajo, el éxito y la competencia. Finalmente, las políticas económicas son muy importantes. Buenas políticas económicas -vale decir políticas que enfatizan la eficiencia, la estabilidad y la credibilidad-, constribuyen al logro de una tasa de productividad más alta, y por tanto, a un mayor crecimiento.

Un análisis de estos factores determinantes del crecimiento para el caso latinoamericano sugiere que la mayoría de los países de la región adolecen de importantes limitaciones. Para empezar, lograr tasas de acumulación de capital elevadas -similares a las de Asia oriental, por ejemplo-exige tasas de ahorro nacional relativamente altas. En la mayoría de los países de América Latina, sin embargo, las tasas de ahorro continúan siendo bajas, y apenas bordean el 20\% del PIB. Además, limitaciones en el funcionamiento de los mercados de capital locales -y muy especialmente las dificultades para financiarse que enfrentan las empresas medianas y pequeñas-hacen que el rendimiento de la inversión sea inferior a su potencial.

La utilización de la mano de obra -la segunda fuente de crecimiento- también 
enfrenta problemas serios prácticamente en toda la región. Las regulaciones del mercado del trabajo -regulaciones bien intencionadas, pero costosas-impiden un crecimiento acelerado del empleo. Además, la participación femenina en la fuerza de trabajo es mucho menor a la de países de nivel de desarrollo equivalente. Más aún, la calidad del sistema educativo latinoamericano deja mucho que desear, lo que se traduce en una acumulación de capital humano inadecuado. Los resultados alcanzados por los países latinoamericanos en las pruebas internacionales que miden la calidad de la educación -pruebas como TIMSS y PISA- son francamente deplorables.

\section{Las regulaciones del mercado de trabajo impiden el crecimiento acelerado del empleo.}

Finalmente, la tasa de crecimiento de la productividad ha sido, tradicionalmente muy baja y nada indica que esta realidad vaya a cambiar. De hecho, en la mayoría de los países latinoamericanos, la calidad de la educación superior-un determinante clave de la capacidad de innovar- es relativamente baja. Además, la relación entre el sector privado y las instituciones de investigación científica y tecnológica es pobre. De acuerdo con la clasificación elaborada por el Suplemento de Educación Superior del prestigioso periódico londinense The Times de 2004, la calidad de la investigación y la enseñanza en las universidades latinoamericanas es mediocre, por decir lo menos. Esta es una clasi- ficación que se basa en el número de publicaciones en revistas académicas, las patentes obtenidas por las distintas universidades, y la frecuencia con la que los profesores son citados en publicaciones especializadas. El estudio del Times cubre varias categorías, incluyendo ciencias, ingeniería y tecnología de la información, y biotecnología (ver http://www.thes.co.uk). Según esta clasificación, los primeros puestos en cada una de las categorías científicas están dominados por universidades anglosajonas -la Universidad de California, en Berkeley; Harvard; el MIT; Cal Tech; Stanford; Cambridge y Oxford-, lo que no es del todo sorprendente. Pero lo que es verdaderamente interesante es que entre las 100 mejores universidades del mundo hay una serie de instituciones de países emergentes, pero ninguna de América Latina. En ciencias, China tiene siete universidades entre las 100 mejores; Israel cuenta con tres; Corea con dos; India con una; y Singapur con una. La cosa es aún más impresionante en la categoría de «ingeniería y tecnología de la información»: entre las diez mejores universidades del mundo hay tres de países emergentes (India, Singapur y China), y varias otras se ubican en los lugares siguientes (pero ninguna latinoamericana). Y en «biotecnología, $\gg$ varios países emergentes tienen universidades ubicadas entre las 100 mejores. Entre ellos destacan China, India, Singapur, Israel, Turquía, Tailandia. Solo en esta categoría aparece una universidad de la región (Brasil) entre los lugares más bajos. Pero esto no es todo, los países avanzados productores de commodities, como Nueva Zelandia, Australia y 
Finlandia, tienen varias universidades ubicadas entre las mejores del mundo, especialmente en el área de la biotecnología. Sus programas ponen en contacto a científicos con distintas empresas, lo que permite desarrollar nuevos y mejores productos de exportación.

El escepticismo reciente respecto de las bondades de las reformas, sumadas a la impaciencia del público y a las tendencias neopopulistas de muchos políticos, indican que en la mayoría de los países de la región no habrá -o al menos no habrá en el mediano plazo- la voluntad ni la capacidad política para poner en ejecución medidas que ataquen las debilidades económicas discutidas más arriba.

El crecimiento económico es un proceso complejo en el que influyen en forma no-lineal los factores antes examinados. La contribución específica de cada factor varía en el tiempo y es específica para cada país. Tanto las instituciones como la cultura y la política económicalos tres factores meta-determinantes del crecimiento discutidos anteriormenteafectan los resultados en cada país. Una característica importante de la transición exitosa hacia una tasa de crecimiento elevada es que se requiere un «detonante» que inicie el proceso. Habitualmente este detonante -que puede ser el fin de una crisis, o de un conflicto armado, o de un proceso de inestabilidad, o la firma de un tratado internacional- da lugar a un fuerte aumento de la tasa de productividad. A su vez, esto genera mayores rentabilidades para la inversión y una afluencia de capital extranjero. Ambos factores elevan el crecimiento económico y la tasa de ahorro nacional. Cuando aumenta el ahorro, la inversión vuelve a crecer, dándole nuevo impulso al crecimiento. A estas alturas de un proceso exitoso, los incrementos de la productividad generados por el «detonante» inicial empiezan a disminuir. Sin embargo, la mayor inversión producida permite sostener un crecimiento elevado, hasta que un nuevo detonante genere un nuevo salto en la productividad. Este círculo virtuoso tiene mayores probabilidades de producirse y mantenerse en países que cuentan con instituciones fuertes y políticas que promueven la competencia. Hoy en día en América Latina, Chile parece ser el único país que ha logrado desarrollar este mecanismo de crecimiento autosostenido.

\section{Conclusiones}

En los últimos cuarenta años América Latina ha experimentado enormes cambios económicos. Las políticas proteccionistas y desarrollistas de los años sesenta y setenta han sido reemplazadas por la apertura y un sistema de mercado. Sin embargo, la región continúa enfrentando serias falencias en materia de crecimiento económico. El análisis presentado en este artículo sugiere que el futuro económico de la región se caracterizará por una ausencia de grandes crisis, y por un crecimiento económico más bien mediocre. 